\title{
Very Forward Calorimetry at the FCC
}

\section{J. Wetzel ${ }^{a, *}$ and Y. Onel ${ }^{a}$}

${ }^{a}$ The University of Iowa, 203 Van Allen Hall, Iowa City, USA

E-mail: james-wetzel@uiowa.edu, yasar-onel@uiowa.edu

The success of any particle detector at a collider experiment depends on its ability to measure both the trajectories and the energies of particles exiting the interaction point. Especially important and difficult is measuring the trajectories and energies of particles in the very forward region where particles that exit the detector have very shallow angles compared to the beam line. The challenge of measuring these particles with high precision is related to the high radiation this area receives, making robust instrumentation a challenge. This region becomes more important with increasing beam energy. We propose a radiation hard, precise, and highly resolved tracking calorimeter that addresses all of these challenges. The proposed design uses highly segmented radiation resistant quartz tiles directly coupled to replaceable radiation resistant photomultiplier tubes. Charged particles entering the quartz array will generate Cherenkov light proportional to their energy, and this light will be measured with photomultiplier tubes. Tracks can be drawn between coinciding signals, and trajectories measured. Neutral particles will leave no initial track in the quartz, but layers of absorber between the quartz tiles will initiate a shower, making it possible to measure all types of particles and energies using this detector. Neural nets can be used to identify particles and tracks. Our simulations show that this detector has excellent tracking, excellent electromagnetic energy resolution, and excellent hadronic energy resolution. Its radiation tolerant materials make it well suited for high radiation environments in addition to its good performance as a calorimeter.

\footnotetext{
*** The European Physical Society Conference on High Energy Physics (EPS-HEP2021), ***

*** 26-30 July 2021 ***

*** Online conference, jointly organized by Universität Hamburg and the research center DESY ***
}

\footnotetext{
*Speaker
} 


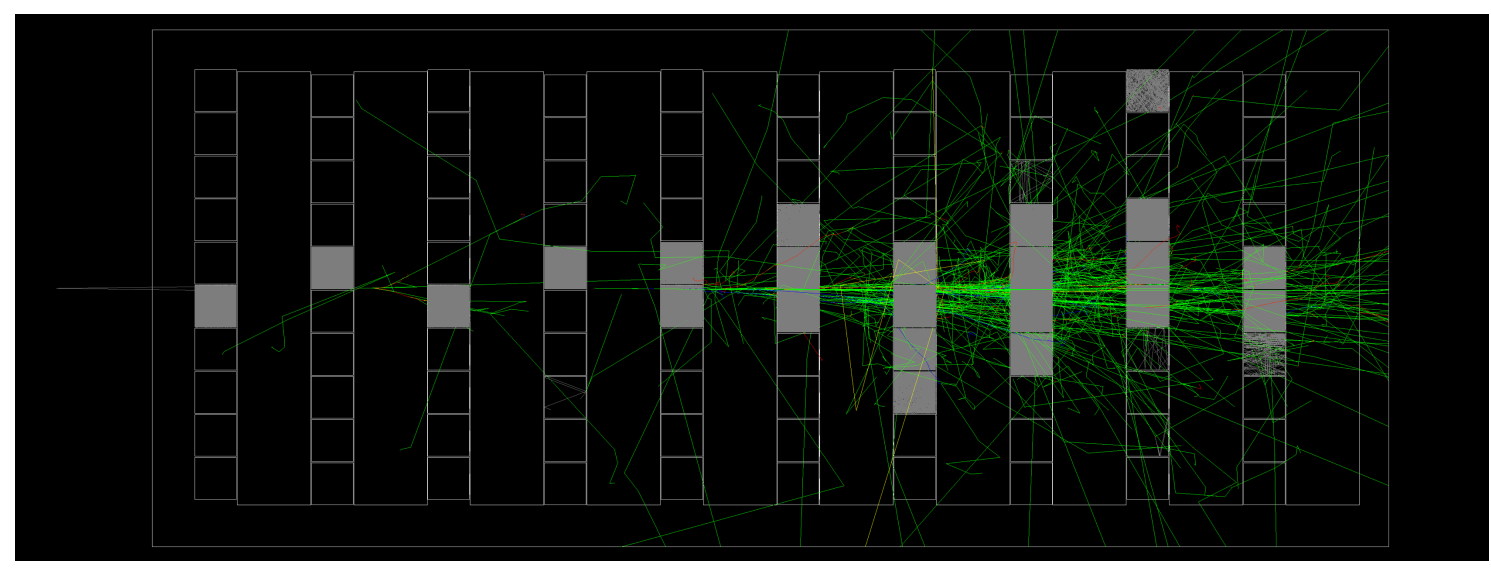

Figure 1: This is a side view projection of the proposed detector concept as constructed in GEANT4. An electron is seen entering the center of the detector from the left, producing an electromagnetic shower (green) and Cherenkov photons (gray). The quartz cubes in this diagram are $1 \mathrm{~cm}$ on a side. This model uses SiPMs, not photomultipliers, and models the associated quantum efficiency. The iron layers are one radiation length thick, $1.757 \mathrm{~cm}$. This is not the geometry used for the discussed performance calculations, only shown to exemplify the general configuration and properties.

\section{Introduction}

The current radiation environment generated by the Large Hadron Collider in its experimental halls are already challenging the standard devices and materials used to conduct collider physics research [1] [2]. CERN is currently planning to extend its research program with the addition of a post LHC collider called the Future Circular Collider (FCC) beyond the year 2040. The environment at the FCC is expected to produce unprecedented radiation fields because of its high luminosity design goal. New material and detector technologies and radiation mitigation strategies must be developed to operate successfully at the FCC for any useful period. Quartz as a material, and photomultiplier tubes (PMTS) as a technology both have robust radiation resistance. This paper discusses a nascent concept of using quartz as a Cherenkov radiation [3] generator and either PMTs or SiPMs as a Cherenkov radiation detector to detect and characterize a variety of particles. Simulations with GEANT4 [4] demonstrate promise as a candidate calorimeter and tracking technology.

\section{Design}

Discussed within this paper are two applications of the same general idea. The central detector design concept proposed is a 3D cubic array of quartz tiles coupled to similarly sized PMTs or SiPMs (referred from now on as the active areas) exemplified in Fig. 1. Each individual quartz cube and photodetector is optically isolated from its neighbors. In Fig. 1, layers of iron absorber are placed between the active layers. Each layers is 1 radiation length thick, or $1.757 \mathrm{~cm}$, to measure its performance as an electromagnetic calorimeter. One of the described applications contained within was for the forward region of the CMS experiment at the LHC, where each active PMT layer is sandwiched between 4 radiation lengths of iron absorber. The overall length of the modeled 


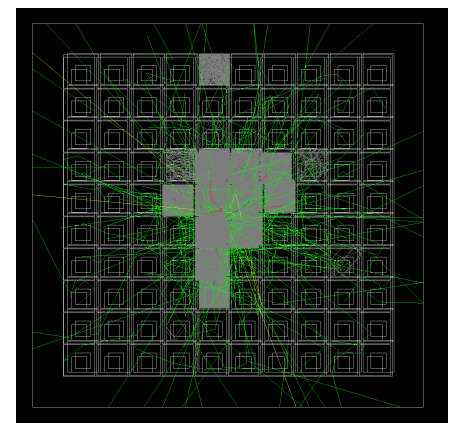

Figure 2: Downstream view of an electron showering with the SiPMs viewable. Note the offset between alternating layers of SiPMs intended to help with precision tracking.
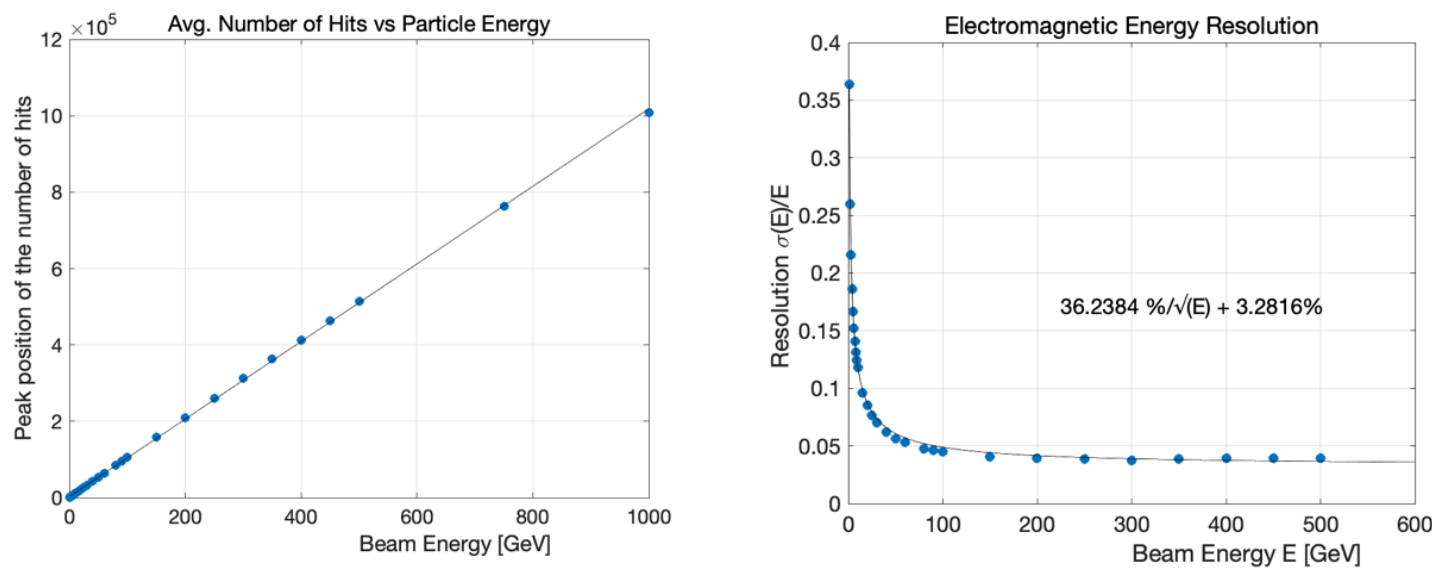

Figure 3: Downstream view of an electron showering with the SiPMs viewable. Note the offset between alternating layers of SiPMs intended to help with precision tracking.

calorimeter is 20 radiation lengths to initiate shower maximum for a $150 \mathrm{GeV}$ electron. This simulation was done to study this concept as a pre-shower detector for the hadronic forward (HF) detector at CMS.

For the FCC, we simulated a large module within GEANT4 of 100 x 100 x 100 quartz cubes, each $1 \mathrm{~cm}$ on a side, with $3 \mathrm{~mm}$ of tungsten between each layer. The active area was $0.5 \times 0.5 \mathrm{~cm}$ SiPMs directly coupled to quartz cubes. An example of this geometry is in Fig. 2.

This design can be extended in any geometry, with any active layer and any absorber layer, or no absorber. Tracks can be found and ECAL modules can seed HCAL modules as seen in Fig. 1.

\section{Performance}

For the combined electromagnetic and hadronic pre-shower calorimeter conceived for supplementing the HF detector at CMS, electromagnetic performance is shown in Fig. 3.

In the FCC conception, we modeled muons to study minimum ionizing particles (MIPs), electrons to measure performance as an electromagnetic calorimeter. These results are in Fig. 4. This concept exhibits good linearity and energy resolution. 

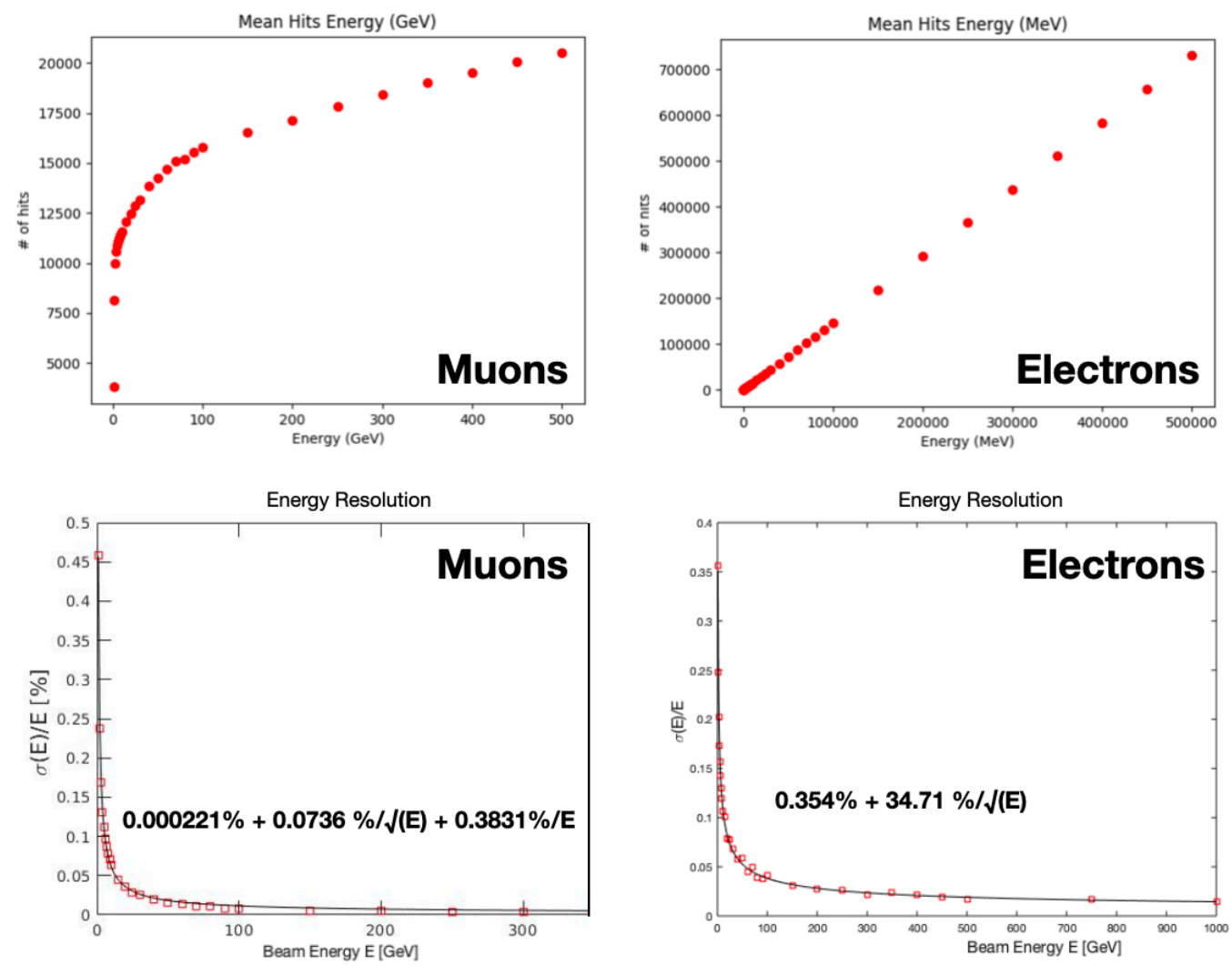

Figure 4: Detector response to MIPs and electrons, directly counting photons vs incident muon energy.

\section{Conclusions}

A highly segmented quartz based Cherenkov detector has great potential for applications at FCC experiments. Its performance is excellence, and the intrinsic radiation tolerance makes it an attractive current technology for use in intense radiation fields.

\section{References}

[1] R. García Alía et al., "LHC and HL-LHC: Present and Future Radiation Environment in the High-Luminosity Collision Points and RHA Implications," in IEEE Transactions on Nuclear Science, vol. 65, no. 1, pp. 448-456, Jan. 2018, doi: 10.1109/TNS.2017.2776107.

[2] P. de Barbaro, V. Epshteyn, "Radiation Damage of the CERN CMS HCAL Scintillator/WLS fiber readout during Run1 and Run2 of the LHC", Technical Report CMS CR-2017/293.

[3] Bolotovskii B M "Vavilov-Cherenkov radiation: its discovery and application" Phys. Usp. 52 1099-1110 (2009)

[4] Agostinelli, Sea, et al. "GEANT4-a simulation toolkit." Nuclear instruments and methods in physics research section A: Accelerators, Spectrometers, Detectors and Associated Equipment 506.3 (2003): 250-303. 